\title{
Characterizing Vascular Connectivity from microCT Images
}

\author{
Marcel Jackowski ${ }^{1}$, Xenophon Papademetris ${ }^{1,2}$, Lawrence W. Dobrucki ${ }^{1,3}$, \\ Albert J. Sinusas ${ }^{1,3}$, and Lawrence H. Staib ${ }^{1,2}$ \\ 1 Departments of Diagnostic Radiology \\ 2 Biomedical Engineering \\ ${ }^{3}$ Medicine (Cardiology), Yale University, New Haven CT 06520
}

\begin{abstract}
X-ray microCT (computed tomography) has become a valuable tool in the analysis of vascular architecture in small animals. Because of its high resolution, a detailed assessment of blood vessel physiology and pathology is possible. Vascular measurement from noninvasive imaging is important for the study and quantification of vessel disease and can aid in diagnosis, as well as measure disease progression and response to therapy. The analysis of tracked vessel trajectories enables the derivation of vessel connectivity information, lengths between vessel junctions as well as level of ramification, contributing to a quantitative analysis of vessel architecture. In this paper, we introduce a new vessel tracking methodology based on wave propagation in oriented domains. Vessel orientation and vessel likelihood are estimated based on an eigenanalysis of gray-level Hessian matrices computed at multiple scales. An anisotropic wavefront then propagates through this vector field with a speed modulated by the maximum vesselness response at each location. Putative vessel trajectories can be found by tracing the characteristics of the propagation solution between different points. We present preliminary results from both synthetic and mouse microCT image data.
\end{abstract}

\section{Introduction}

Methods for the quantification of vascular structure are crucial in a number of domains. While 3D localization and visualization are important, the power of vascular imaging methods lies in quantitative analysis including characterizing and measuring connectivity, level of ramification, segment length as well as crosssectional area and volume. A particularly important application is the study of changes that occur in response to angiogenic therapy which has tremendous potential for treatment in vessel disease and would benefit from non-invasive methods for quantitative evaluation of vasculature growth and remodeling.

$\mathrm{X}$-ray microCT imaging combined with perfused contrast agents provides a robust methodology for evaluation of intact vascular networks [4]. However, the ability to extract and quantify vessels, especially those of smaller diameter, is limited by noise, contrast and extraneous features, such as bone. In CT using contrast agents, vessels appear as relatively bright; in magnetic resonance 
angiography (MRA), they may be dark or bright depending on the technique. They are curved structures of varying width with greatest width at the aorta, generally decreasing in diameter with branching and greater distance from the aorta. The geometry is quite complex making $3 \mathrm{D}$ imaging a necessity $(2 \mathrm{D}$ serial section analysis is only of limited use).

Techniques for filtering curved structures have been applied to vessel images 15|9|6 11, especially using multiscale techniques. In these methods, the Hessian of the gray level intensities is computed at multiple scales. The eigenstructure of the Hessian is determined in order to characterize the local structure (linear, planar or no structure) and if linear, the orientation of the vessel. Methods have been developed for vessel segmentation using thresholding [15], active contours [1], model-based methods 98, expectation maximization [1] as well as level setbased approaches [12]3. Lorigo et al. [12] applied a novel level-set segmentation formulated specifically for 3D curves. Vessel segmentation, however, is prone to errors and may result in discontinuous segments and inclusion of extraneous features, such as bone. Work on tracing vessel trajectories has also been attempted before. Olabarriaga et al. find a minimum cost path through a vesselness image using bidirectional search 14. Flasque et al. [5] track vessels from MRA using a model-based approach, tracking centerlines using a search strategy. Deschamps and Cohen presented a minimal-path method based on the Fast Marching algorithm applied to vessel centerline extraction for virtual endoscopy [2] Lin [10] also investigated extracting minimal paths using an anisotropic version of the Fast Marching method that incorporates orientation and was applied to MRA and fluorescence images.

In this paper, we also focus on extracting vessel trajectories. Our approach is similar to that of Lin [10, by using an anisotropic wavefront evolution method, and like Descoteaux [3] , we also make use of Frangi's vesselness measure. While we are interested in finding the size of extracted vessels, which can be obtained by the vesselness response across scales, we do not use level sets to explicitly reconstruct vessel boundaries. Levels sets for explicit surface reconstruction can easily bleed out into regions with similar vessel intensities. They also have difficulty extracting finer-scale vessels and may result in disconnected vessel segments. In the next section, we describe our vessel tracing approach using a static anisotropic wavefront propagation method combined with a multiscale vessel analysis.

\section{Approach}

In this section, we briefly describe the multiscale vessel localization procedure and how to obtain the vessel likelihood measure [6. Then we describe the wave propagation equation that will drive the front evolution using information from the Hessian matrix as well as the maximum vessel likelihood response across different scales. Finally, we describe how to extract vessel trajectories by tracing the characteristics of the front evolution PDE. An anisotropic version of the static front evolution method is used to build a cost map which will be minimum for pathways in highly oriented structures and with high vesselness measure. 


\subsection{Vessel Filtering}

One way to account for the varying size of vessels is by multiscale analysis. It allows us to detect structures of different sizes according to the scale at which they give maximal response. In order to enhance blood vessels at a particular scale $\sigma$, Frangi [6] designed the filter $V(x, \sigma)$, which is a nonlinear combination of the eigenvalues of the Hessian matrix $\mathcal{H}$ computed at each voxel of the image. This vesselness measure has given reasonable results in the segmentation of blood vessels by other investigators 3 and is also employed here. The scale corresponds to the vessel radius in pixels.

The filter $V$ provides a likelihood value for a structure having a tubular shape. At each scale $\sigma$, the image is first convolved by a 3-D Gaussian with standard deviation equal to that scale in pixels and then the vesselness measure is computed as:

$$
V(x, \sigma)=\left\{\begin{array}{l}
0, \quad \text { if } \lambda_{2}>0 \text { or } \lambda_{3}>0 \\
\left(1-\exp \left(-\frac{R_{A}^{2}}{2 \alpha^{2}}\right)\right) \exp \left(-\frac{R_{B}^{2}}{2 \beta^{2}}\right)\left(1-\exp \left(-\frac{S^{2}}{2 c^{2}}\right)\right), \text { otherwise }
\end{array}\right.
$$

where $R_{A}=\frac{\left|\lambda_{2}\right|}{\left|\lambda_{3}\right|}, \quad R_{B}=\frac{\left|\lambda_{1}\right|}{\sqrt{\left|\lambda_{2} \lambda_{3}\right|}}, \quad S=\sqrt{\lambda_{1}^{2}+\lambda_{2}^{2}+\lambda_{3}^{2}}$ and the eigenvalues are ordered according to their magnitudes as $\left|\lambda_{1}\right| \leq\left|\lambda_{2}\right| \leq\left|\lambda_{3}\right|$. Since the vessels are bright against a darker background in microCT images, the eigenvalues are negative. The quantities $R_{A}, R_{B}$ and $S$ are designed to punish cross-sectional asymmetry, blobness and low energy, respectively. The parameters $\alpha, \beta$ and $c$ are used to tune the sensitivity of the filter to deviations from perfect tubular structures. This measure will yield maximum response at the center of vessels and close to zero outside them. The filter $V$ is applied at different scales in order to detect vessels with different scales. We vary $\sigma$ from $\sigma_{\min }$ to $\sigma_{\max }$, and compute the maximum vessel likelihood across all chosen radii:

$$
V_{\max }(x)=\max _{\sigma_{\min } \leq \sigma \leq \sigma_{\max }} V(x, \sigma) .
$$

\subsection{Front Evolution}

Once the maximum response $V_{\max }$ is computed for all points in the domain, not only will we have a vessel likelihood map but also a map containing the corresponding Hessian matrix $\mathcal{H}_{\max }$ at the maximum scale. From the eigenanalysis of $\mathcal{H}_{\text {max }}$, the eigenvector $\boldsymbol{u}=\boldsymbol{u}_{1}$ which corresponds to the smallest eigenvalue will represent the vessel orientation. In our implementation, we will propagate a wavefront through the vector field defined by $\boldsymbol{u}$. The front will move fastest in regions where its normal vector $\boldsymbol{n}=\frac{\nabla T}{\|\nabla T\|}$ lines up with the vector field and in regions with a high vesselness measure. $T$ represents the time of arrival of the front at each location. Such evolution can be modeled by the following static anisotropic evolution equation:

$$
\|\nabla T\| V_{\max }(x) F(x, \boldsymbol{n})=1, \quad F(x, \boldsymbol{n})=\exp \left(\{\boldsymbol{n} \cdot \boldsymbol{u}\}^{2}\right) .
$$


This propagation equation will evolve a wavefront driven by the directionality of the vector field $\boldsymbol{u}$ but will stop its evolution near the boundary with non-vessel structures, since $V_{\max }$ will be close to zero in these regions. Since the Hessian matrix is indefinite [10, not all eigenvalues have the same sign. Hence, $\mathcal{H}_{\max }$ may not represent an ellipsoid at all times. Instead of using the full Hessian as an ellipsoidal speed profile, we resort to driving our wavefront according to the colinearity between the front normal $\boldsymbol{n}$ and $\boldsymbol{u}$.

Equations such as (3) cannot be correctly solved by isotropic propagation methods, such as the Fast Marching Method. Hence, we employ an iterative approach combined with a Lax-Friedrichs (LF) discretization of our propagation equation. A nonlinear Gauss-Seidel updating scheme is used to solve the equation in terms of neighboring grid points. No minimization is required when updating an arrival time, and thus it becomes very easy to implement.

In order to track vessel segments, we seed the evolution at a point inside the vessel, and let the propagation take place. When the evolution has converged to a solution, it will represent a cost map which can be used to find a minimal cost path representing the vessel trajectory.

\subsection{Vessel Tracking}

Minimum-cost trajectories are determined by the characteristic curves of the respective partial differential equation [137. In our propagation model, the gradient $\nabla T$ of the solution will not point to the minimum-cost path, since our equation is anisotropic and the speed profile is not circular. One must explicitly calculate the appropriate characteristic directions of the obtained arrival times solution at every point.

A generic first-order PDE with $m$ independent variables can be written as:

$$
H\left(x_{i}, T, p_{i}\right)=0, \text { where } p_{i}=\partial T / \partial x_{i}, i=1, \ldots, m
$$

where $T$ is a function of each of the independent variables $x_{i}$. In our case, $T$ represents the arrival times of the wavefront. The characteristic vector $\boldsymbol{a}$ can be obtained via Charpit's equations [13 and is defined as $a_{i}=\partial H / \partial p_{i}$. Our wavefront evolution equation (3) can be written in generic form by discarding its dependence on location $x$ :

$$
H=\sqrt{p_{1}^{2}+p_{2}^{2}+p_{3}^{2}} V_{\max } F\left(p_{1}, p_{2}, p_{3}\right)-1=0
$$

By differentiating $H$ with respect to $p_{1}, p_{2}$ and $p_{3}$, we find the characteristic vector $\boldsymbol{a}$. Because the speed function $F$ is a function of the gradient $\nabla T$, the characteristic vector $\boldsymbol{a}$ does not necessarily coincide with the gradient. Therefore, one must integrate $\frac{d X}{d t}=-\boldsymbol{a}$ instead of $\frac{d X}{d t}=-\nabla T$ to obtain the minimum-cost path, $X$.

\section{Experimental Results}

In order to evaluate our vessel tracing method, we first applied it on a synthetic dataset obtained from the Laboratory of Mathematics in Imaging at Harvard 
University 9]. Figure 17 depicts the Y-junction vessel model which contains 2 segments of different radii (1 and 3 pixels). They have Gaussian intensity profiles with a maximum intensity value of 100 at their centerlines. Fig. 1b shows the corresponding ridges (centerlines) from the model. The centerlines are assumed to be the true trajectories. We investigated how close the resulting trajectories were to the true centerlines, with and without noise. Gaussian noise with $\sigma^{2}=10,20,40$ was added to the original image. The maximum vesselness measure $V_{\max }$ and the respective Hessian $\mathcal{H}_{\max }$ were then computed at two different scales, 1 and 3 pixels to match the exact model dimensions (Fig. 11-e). Parameters $\alpha, \beta$ and $c$ were set to $0.5,0.5$ and half the maximum Frobenius norm of the Hessian matrices, respectively, according to [6]. Next, we employed our wave propagation technique by first selecting a seed point for propagation (see cross-hairs in Fig. 17a). After propagation reached convergence, we traced the centerlines from the extreme points of the two branches (Figs. 1f-h) and compared the results to the true trajectories (Fig. 1b).

As can be seen in Figs. 1f-h, the technique is robust enough to recover the trajectories embedded in very noisy backgrounds. This is partly due to the Gaussian

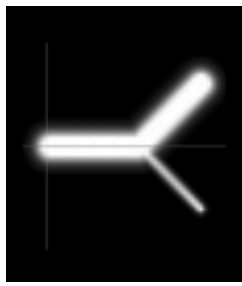

(a)

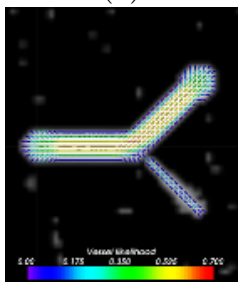

(c)

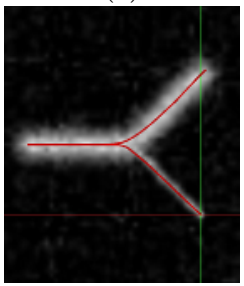

(f)

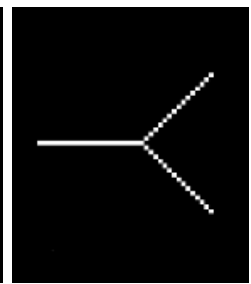

(b)

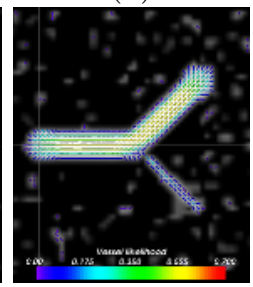

(d)

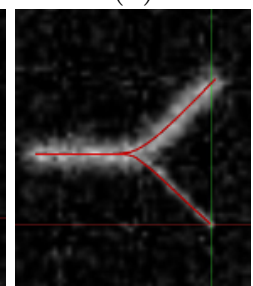

(g)

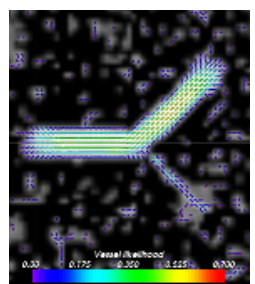

(e)

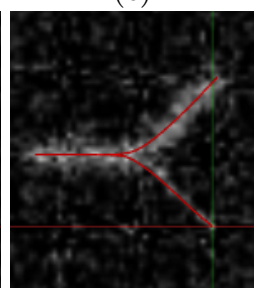

(h)

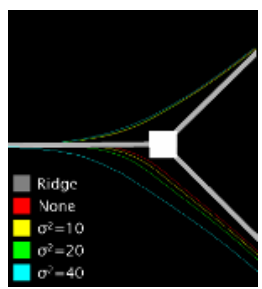

(i)

Fig. 1. (a) Synthetic dataset. (b) Corresponding centerlines. Second row: Vector field $\boldsymbol{u}$ at different noise levels; (c) $\sigma^{2}=10$, (d) $\sigma^{2}=20$, (e) $\sigma^{2}=40$. Third row: Resulting trajectories at different noise levels. (i) Close-up at branching point. 
smoothing that is applied at each scale when determining the maximum vesselness response. Figure 1 shows a close-up view of the branching point in the model. The maximum disparities between the centerline (shown in gray) and the resulting trajectories were found to be at the center of the branching point (shown as a white square in Fig. 11). This is because our speed term does not currently follow the ridges of the vessels, only their directionality. Therefore, the minimum-cost trajectory may not pass exactly through the true center of the intersection. For the larger branch (3 pixels in radius), the maximum error averaged $\approx 1$ voxel, with and without added noise (no-noise trajectory shown in red). The largest error was obtained for the smaller size branch, as large levels of noise destroyed a lot of its structure and subsequent smoothing altered its final

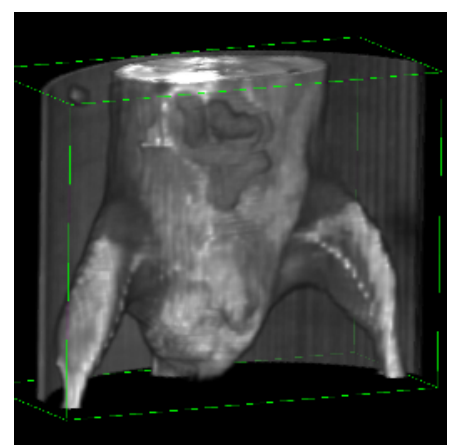

(a)

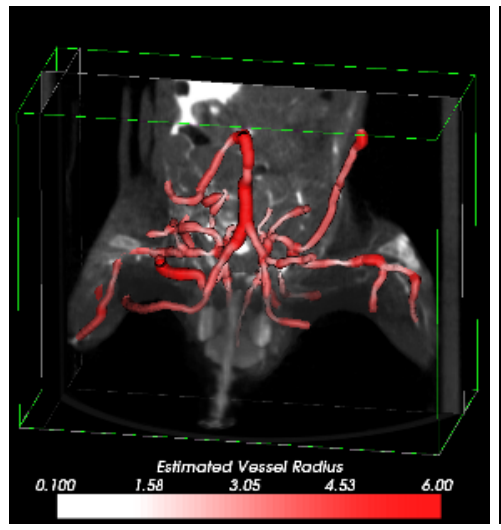

(c)

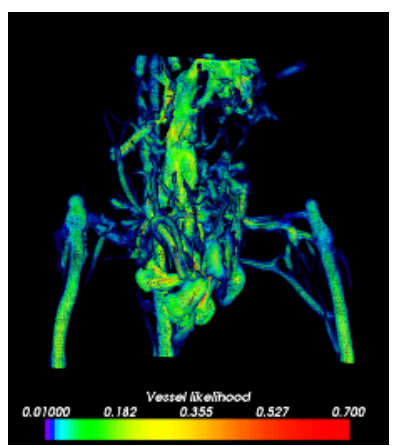

(b)

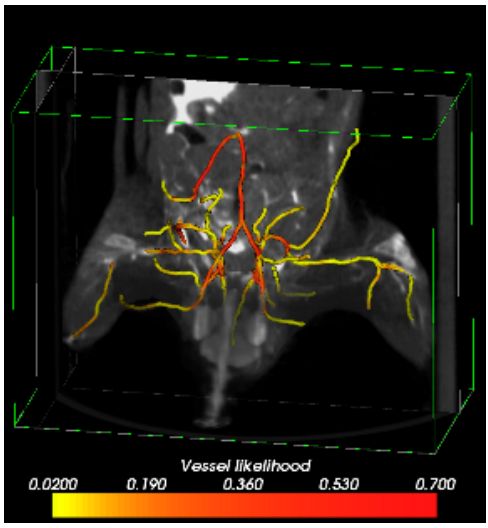

(d)

Fig. 2. (a) Volume rendering of the CT dataset depicting the lower body of a mouse. (b) Three-dimensional view of the vector field $\boldsymbol{u}$ colored by the vesselness measure. Both bones and vessels can be seen. (c) Reconstruction of the major vessels connecting to the aorta shown with volume rendering. Vessels are colored according to their estimated size. (d) Reconstructed vessels colored according to their maximum vesselness measure. Vessels located in the inferior half of the volume were partially obscured for better spatial perception. 
shape. A maximum error of $\approx 1.7$ voxels resulted at $\sigma^{2}=40$. The corresponding errors for no-noise was 0.79 voxels, with noise $\sigma^{2}=10$ was $0.92, \sigma^{2}=20$ was 1.13 and $\sigma^{2}=40$ reached 1.73 voxels. Despite the discrepancies from the true centerline, the technique presented here allows for fine vessel extraction in the presence of significant noise, and will not result in disconnected structures, as may occur with other level set-based techniques.

To evaluate our method on a real image, an ex-vivo mouse microCT dataset using barium-sulfate contrast was acquired at a resolution of $50 \times 50 \times 100 \mu \mathrm{m}$. (Figure 2a). After cropping, the resulting image size was $291 \times 131 \times 226$. A mean curvature-based smoothing was first applied to eliminate undesired background noise while preserving edge information. Multiscale analysis was performed in 10 uniformly sampled Gaussian scales ranging from 0.5 to 5 voxels. The maximum vesselness measure and the corresponding Hessian $\mathcal{H}_{\text {max }}$ was computed at each location. Figure $2 \mathrm{~b}$ shows the vector field $\boldsymbol{u}$ after diagonalizing $\mathcal{H}_{\text {max }}$. The vector field is colored according to the vesselness measure at each point. Both vessels and bones can be seen. A seed point was placed inside the aorta and a wavefront was propagated in the vector field $\boldsymbol{u}$. In order to initialize tracking, 45 points were selected manually by picking the center of vessels at different planes. Vessel trajectories were then traced back into the aorta. Estimates of radius and vesselness measures were calculated at every point on the trajectories.

Figure 2r shows the resulting trajectories as tubular structures and colored according to their estimated radius. Because a discrete number of scales were employed, tubes representing the vessels did not change in size continuously. With a more continuous scaling, better radii estimates can be determined. Radii were determined by the scale in which the vesselness measure was maximum. Figure $2 \mathrm{~d}$ shows the extracted vessels colored according to their maximum vesselness measure.

\section{Conclusions}

An anisotropic front propagation method was described for determining vascular pathways in mouse microCT images. Using multiscale analysis, the maximum vesselness measure was computed at every point and the corresponding Hessian matrix was recorded. A wavefront was propagated in the vector field defined by the smallest eigenvector of the Hessian matrix. The propagation speed was defined by the colinearity between front normal and the smallest eigenvector of the Hessian. The front stopped at regions of very low vesselness. Using the characteristic vector of the propagation solution, the technique was able to recover trajectories in both synthetic and real microCT data.

Results on mouse CT datasets were presented where major vessels were recovered and reconstructed. Vessel trajectories were fit with tubular structures with a radius corresponding to the scale in which they were detected. Further investigation will be done on new forms of the evolution PDE for tracking the ridges. We will also fit ellipses instead of circles for the vessel reconstruction by using the eigenvalue magnitudes from the Hessian matrix. In addition, we will 
investigate an automated approach for initializing the vessel tracing which will enable us to reconstruct the entire vessel tree and allow for its quantification with minimum manual intervention. Features such as connectivity, level of ramification, segment length as well as cross-sectional area and volume can then be calculated. These measures may ultimately improve quantitative diagnosis and allow the measurement of change due to disease or therapy.

\section{References}

1. A. Chung and J. A. Noble. Statistical 3D vessel segmentation using a Rician distribution. In MICCAI, pages 82-89. LNCS 1679, Springer, 1999.

2. D. Deschamps and L. D. Cohen. Fast extraction of minimal paths in 3d images and applications to virtual endoscopy. Medical Image Analysis, 5(4), Dec 2001.

3. M. Descoteaux. A multi-scale geometric flow for segmenting vasculature in MRI: Theory and validation. Master's thesis, School of Computer Science, McGill University, Montréal, June 2004.

4. C. L. Duvall, W. R. Taylor, D. Weiss, and R. E. Guldberg. Quantitative microcomputed tomography analysis of collateral vessel development after ischemic injury. Am. J. Physiol. Heart. Circ. Physiol., 287(1):H302-310, 2004.

5. N. Flasque, M. Desvignes, J. Constans, and M. Revenu. Acquisition, segmentation and tracking of the cerebral vascular tree on 3D magnetic resonance angiography images. Medical Image Analysis, 5(3):173-183, Sep 2001.

6. A. F. Frangi, W. J. Niessen, K. L. Vincken, and M. A. Viergever. Multiscale vessel enhancement filtering. In MICCAI, pages 130-137. LNCS 1496, Springer, 1998.

7. F. John. Partial Differential Equations, volume 1. Springer, 4th edition, 1982.

8. K. Krissian, R. Kikinis, and C. Westin. Algorithms for extracting vessel centerlines. Technical Report 0003, Department of Radiology, Brigham and Women's Hospital, Harvard Medical School, Laboratory of Mathematics in Imaging, September 2004.

9. K. Krissian, G. Malandain, N. Ayache, R. Vaillant, and Y. Trousset. Model-based detection of tubular structures in 3D images. Comp. Vision and Image Understanding, 80(2):130-171, 2000.

10. Q. Lin. Enhancement, Extraction and Visualization of $3 D$ Volume Data. $\mathrm{PhD}$ thesis, Dept. of Electrical Engineering, Linköping University, Sweden, 2003.

11. C. Lorenz, I.-C. Carlsen, T. M. Buzug, C. Fassnacht, and J. Weese. A multi-scale line f ilter with automatic scale selection based on the Hessian matrix for medical image segmentation. In Scale-Space Theories in Computer Vision, pages 152-163. LNCS 1252, Springer, 1997.

12. L. M. Lorigo, O. D. Faugeras, W. E. Grimson, R. Keriven, R. Kikinis, A. Nabavi, and C. F. Westin. CURVES: curve evolution for vessel segmentation. Medical Image Analysis, 5(3):195-206, Sep 2001.

13. J. Ockendon, S. Howison, A. Lacey, and A. Movchan. Applied Partial Differential Equations. Oxford University Press, revised edition, 2003.

14. S. D. Olabarriaga, M. Breeuwer, and W. J. Niessen. Minimum cost path algorithm for coronary artery central axis tracking in CT images. In R. E. Ellis and T.M. Peters, editors, MICCAI, pages 687-694, Berlin, 2003. LNCS 2879, Sprin ger.

15. Y. Sato, S. Nakajima, N. Shiraga, H. Atsumi, S. Yoshida, T. Koller, G. Gerig, and R. Kikinis. Three-dimensional multi-scale line filter for segmentation and visualization of curvilinear structures in medical images. Medical Image Analysis, 2(2):143-168, 1998. 